\title{
Multiphoton Autofluorescence and Second-Harmonic Generation Imaging of the Ex Vivo Porcine Eye
}

\author{
Sbu-Wen Teng, ${ }^{1}$ Hsin-Yuan Tan, ${ }^{2,3}$ Ju-Li Peng, ${ }^{4}$ Huei-Hsing Lin, ${ }^{4}$ Ki Hean Kim, ${ }^{5}$ Wen Lo, ${ }^{1}$ \\ Yen Sun, ${ }^{1}$ Wei-Chou Lin, ${ }^{6}$ Sung-Jan Lin, ${ }^{3,7}$ Sbiou-Hwa Jee, ${ }^{7,8}$ Peter T. C. So, ${ }^{5}$ and \\ Chen-Yuan Dong ${ }^{1}$
}

Purpose. The purpose of this work was to demonstrate the use of the combined imaging modality of multiphoton autofluorescence and second-harmonic generation (SHG) microscopy in obtaining spectrally resolved morphologic features of the cornea, limbus, conjunctiva, and sclera in whole, ex vivo porcine eyes.

Methods. The 780-nm output of a femtosecond, titanium-sapphire laser was used to induce broadband autofluorescence $(435-700 \mathrm{~nm})$ and SHG $(390 \mathrm{~nm})$ from various regions of the surface of ex vivo porcine eyes. A water-immersion objective was used for convenient imaging of the curved surface of the eye.

RESults. Multiphoton autofluorescence was useful in identifying cellular structures of the different domains of the ocular surface, and the SHG signal can be used to resolve collagen organization within the cornea stroma and sclera of ex vivo porcine eyes.

Conclusions. Multiphoton autofluorescence and SHG microscopy have been demonstrated to be an effective technique for resolving, respectively, the cellular and collagen structures within the ocular surface of ex vivo porcine eyes. SHG imaging resolved the difference in structural orientations between corneal and sclera collagen fibers. Specifically, the corneal collagen is organized in a depth-dependent fashion, whereas the scleral collagen is randomly packed. Because this technique does not require histologic preparation procedures, it has the potential to be applied for in vivo studies with minimal disturbance to the eye. (Invest Ophthalmol Vis Sci. 2006;47: 1216-1224) DOI:10.1167/iovs.04-1520

From the ${ }^{1}$ Microscopic Biophysics Laboratory, Department of Physics, the ${ }^{3}$ Institute of Biomedical Engineering, College of Medicine and College of Engineering, and the ${ }^{4}$ Department of Life Science, National Taiwan University, Taipai, Taiwan; the Departments of ${ }^{6} \mathrm{~Pa}-$ thology, and ${ }^{7}$ Dermatology, National Taiwan University Hospital, Taipei Taiwan; the ${ }^{8}$ Department of Dermatology, College of Medicine, National Taiwan University Hospital, Taipei, Taiwan; the ${ }^{2}$ Department of Ophthalmology, Chang Gung Memorial Hospital, Linko, Taiwan; and the ${ }^{5}$ Department of Mechanical Engineering, Massachusetts Institute of Technology, Cambridge, Massachusetts.

Supported by Grant NSC 93-3112-B-002-033 from the National Science Council, Taiwan.

Submitted for publication December 25, 2004; revised March 16 and April 24, 2005; accepted January 11, 2006.

Disclosure: S.-W. Teng, None; H.-Y. Tan, None; J.-L. Peng None; H.-H. Lin, None; K.H. Kim, None; W. Lo, None; Y. Sun, None; W.-C. Lin, None; S.-J. Lin, None; S.-H. Jee, None; P.T.C. So, None; C.-Y. Dong, None

The publication costs of this article were defrayed in part by page charge payment. This article must therefore be marked "advertisement" in accordance with 18 U.S.C. $\$ 1734$ solely to indicate this fact.

Corresponding author: Chen-Yuan Dong, Microscopic Biophysics Laboratory, Department of Physics, National Taiwan University, Taipei 106, Taiwan; cydong@phys.ntu.edu.tw.
$\mathrm{T}$ he ability to acquire structural information is of key significance in the physiological studies and disease diagnosis of the eye. Various structures within the ocular surface are of structural and functional significance. Morphologically, it is known that the cornea is covered with epithelium followed by stroma, which is composed mainly of collagen fibers. At the boundary of the cornea and sclera lies the limbus, which contains stem cells responsible for maintaining the corneal epithelium. The outer edge of the ocular surface is composed of sclera, which has a composition similar to the cornea in that it contains collagen fibers, and is covered with conjunctiva. However, unlike corneal collagen fibers, scleral collagen is not well organized into the striation pattern responsible for the cornea transparency. ${ }^{1}$ For many ophthalmic applications, the ability for physiological studies and disease diagnosis of the eye hinges on the ability to visualize the morphologic features of the ocular surface.

As in many areas of biology and medicine, ophthalmology has depended heavily on traditional histologic procedures in probing eye morphology. Although these procedures are invaluable in providing detailed structural information on the eye at the tissue and cellular levels, there are several drawbacks. First, the morphologic artifacts associated with the fixation, labeling, and processing procedures used in histology are always of concern. In addition, histologic processing represents the end of specimen vitality. Observing physiological dynamics under in vivo conditions is impossible after fixation of the sample. With advances in optical imaging technology, the limitations imposed by histologic procedures are now being overcome. For example, optical coherence tomography (OCT), an imaging technique that depends on the interference of light reflected from layers within the eye has been demonstrated to be useful for ophthalmic imaging. ${ }^{2-4}$ In addition, confocal microscopy has been useful for in vivo ophthalmologic observations with applications in disease diagnosis., ${ }^{5,6}$

A third promising imaging modality is multiphoton microscopy. Based on the nonlinear excitation of fluorescent molecules or the induction of harmonic generation (HG), multiphoton microscopy has been widely applied to different areas of the life sciences, including neurobiology, development biology, oncology, transdermal delivery, immunology, hepatology, and deep-tissue imaging. ${ }^{7-20}$ In addition, NADP $(\mathrm{H})$ autofluorescence and collagen second-harmonic generation (SHG) have been applied to cornea imaging. ${ }^{21,22}$ However, to the best of our knowledge, multiphoton imaging has been applied neither to explore cornea structures at a large geometric scale, nor to the investigation of other key features of the ocular surface.

Several advantages enable multiphoton microscopy to be a promising imaging modality in ophthalmology. First, the high photon flux needed to induce nonlinear optical response from the specimen limits sample excitation to the focal spot. Because most fluorescence or harmonic generating signals are excluded from off-focal volume, optical imaging obtained by 


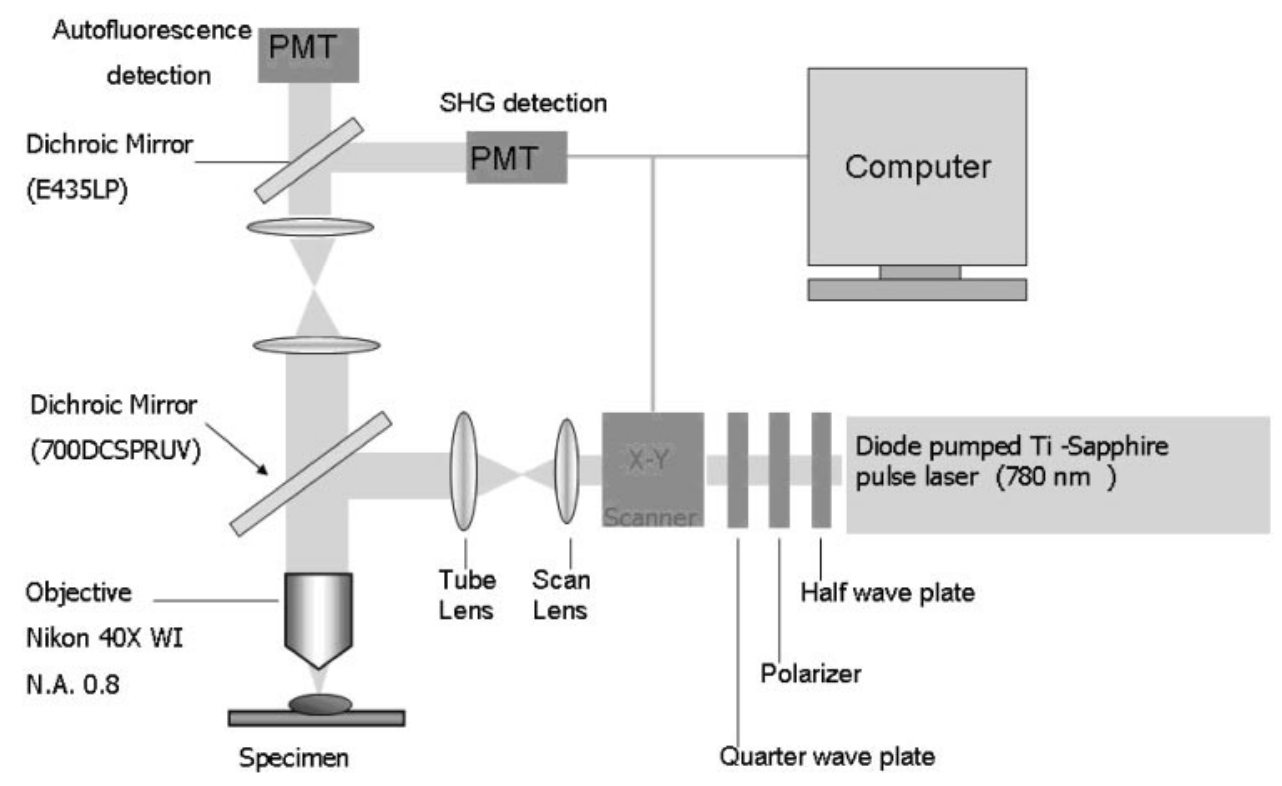

FigURE 1. A multiphoton autofluorescence and SHG microscope.

scanning the pointlike multiphoton volume can generate images with excellent axial depth description and high contrast. Furthermore, the pointlike excitation volume limits specimen photodamage to the focus. This feature enables sample longevity to be achieved. Finally, the near-infrared excitation wavelengths used in multiphoton microscopy are absorbed and scattered less by the tissues than is the ultraviolet or visible radiation used in standard single-photon excitation microscopy. As a result, multiphoton microscopy can be used to achieve in-depth bioimaging without invasive histologic procedures. $^{7,8}$

In ophthalmology, a high-contrast, high-resolution, and large-area imaging technique with the potential of in vivo applications is of immense value in physiological studies and disease diagnosis. Although multiphoton imaging remains to be within the realm of surface tissue imaging, this approach can be readily applied to the ocular surface for obtaining images with subcellular resolution. To begin with, changes to the corneal structures are important in vision correction procedures. In laser in situ keratomileusis (LASIK) or conductive keratoplasty, the ability to monitor structural change of cornea collagen is vital in assessing the success of these procedures. $^{23-26}$ Although histologic procedures are available to monitor changes to the cornea, the transparent nature of the cornea prevents its internal structures to be analyzed easily. ${ }^{27}$ Although x-ray diffraction techniques have added valuable information to the organization of collagen fibers in the cornea, ${ }^{28-31}$ a direct visualization technique to obtain structural information about the cornea in intact eyes would be of immense value in clinical evaluation of corneal states, and multiphoton collagen imaging offers such possibilities. ${ }^{20-22}$ In addition, the limbus, the junctional structure separating the transparent cornea and opaque sclera, is important in maintaining corneal epithelial tissues. Structurally, the limbus contains stem cells that differentiate into corneal epithelium. For ophthalmologists, the ability to monitor the physiological states within the limbus without histologic fixation procedures has potential application in tissue engineering. ${ }^{32,33}$ Specifically, if multiphoton microscopy can be used to image the limbal epithelium, the spectral separation of cellular autofluorescence from the SHG signal of the surrounding cornea collagen fibers will enable high-contrast imaging of the limbus to be achieved. Finally, because the conjunctiva and sclera are respectively organized into connective tissues of the outer edges of the eye, one can anticipate multiphoton autofluorescence and SHG imaging to be useful in spectrally resolving the structures of the sclera from the cornea. In vivo imaging of the sclera is invaluable in assessing tissue states in clinical settings. For example, thermal treatment of the sclera has been used for treatment of ocular melanoma. ${ }^{34}$ However, the ideal scenario would be the eradication of the tumor mass without damaging the surrounding connective tissues. Because the SHG signal is sensitive to the thermal denaturation of collagen fibers, a potential application of SHG imaging is to assess the degree of thermal damage to the sclera without histologic procedures. ${ }^{35}$ Finally, the change of autofluorescence in diabetic patients and diseases such as corneal and conjunctival tumors are potential imaging targets of multiphoton microscopy. ${ }^{36-38}$

\section{Materials ANd Methods}

\section{Multiphoton Microscopy Instrumentation}

The multiphoton microscopy instrument used in this study is similar to a set-up described previously. ${ }^{15} \mathrm{~A}$ commercial upright microscope (E800; Nikon, Tokyo, Japan) was modified into a custom-built multiphoton instrument. A diode-pumped solid-state (DPSS)-pumped (Millennia X; Spectra Physics, Mountain View, CA) titanium-sapphire (ti-sa) laser (Tsunami; Spectra Physics) capable of producing $80-\mathrm{MHz}$, femtosecond laser pulses in the spectral range of 700 to $1000 \mathrm{~nm}$ was used as the excitation source. For our experiments, the 780-nm output of the ti-sa laser was used to induce autofluorescence and SHG from the porcine eye specimens. Before entering the microscope, the excitation laser is guided toward an $x-y$ scanning system (Model 6220; Cambridge Technology, Cambridge, MA) that steers the laser source two dimensionally. At the entrance of the upright microscope, the laser is beam expanded and then reflected by a dichroic mirror (700DCSPRUV; Chroma Technology, Brattleboro, VT) into the back aperture of a water-immersion objective (Fluor $40 \times$, NA 0.8 ; Nikon). The average power at the sample is $16 \mathrm{~mW}$, and the autofluorescence and SHG signals generated are collected by the same objective and passed through the dichroic onto the photodetectors. At $16 \mathrm{~mW}$ of average laser power, we did not observe significant structural damage to the imaged ocular tissues. Our results imply that multiphoton microscopy has the potential to be developed into an in vivo imaging technique in 

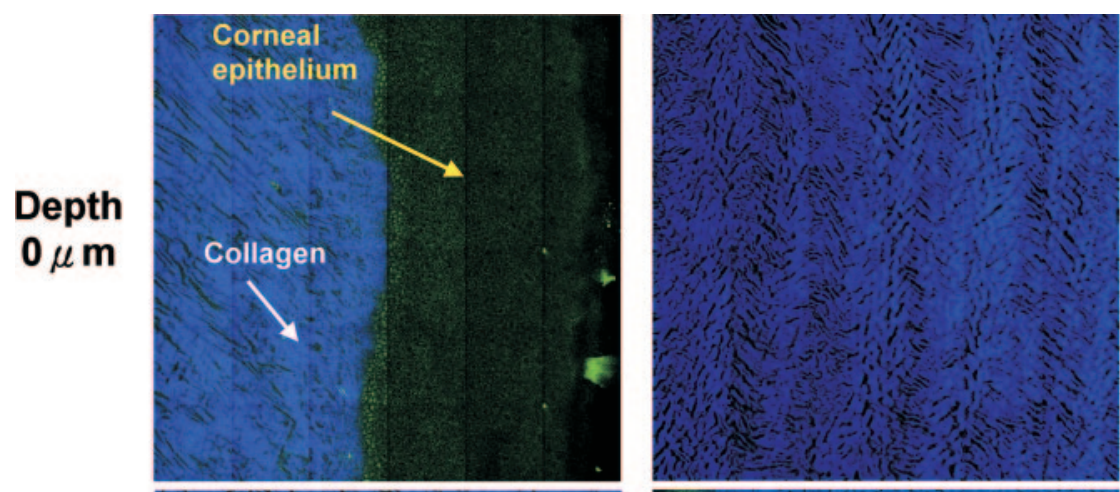

\section{$800 \mu \mathbf{m}$}
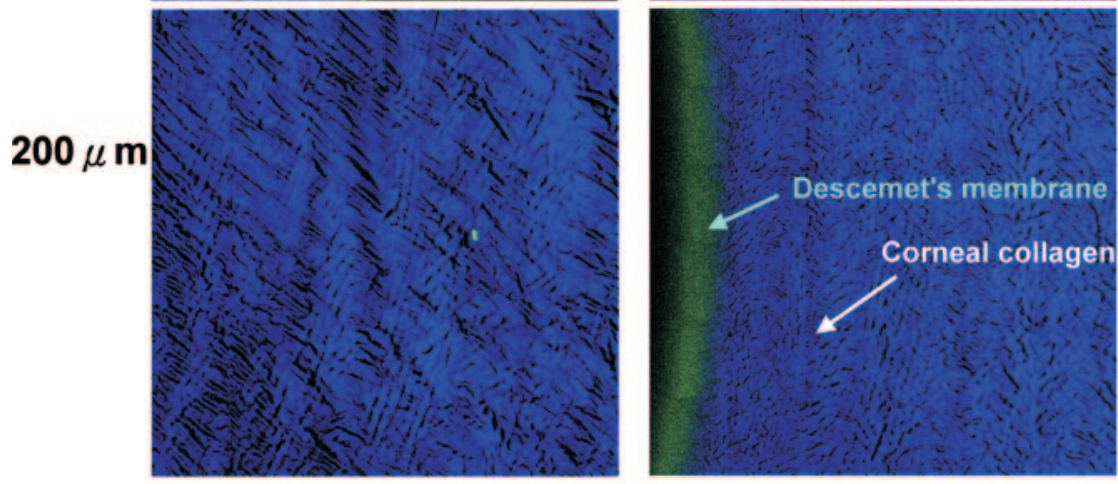

\section{$862 \mu \mathbf{m}$}
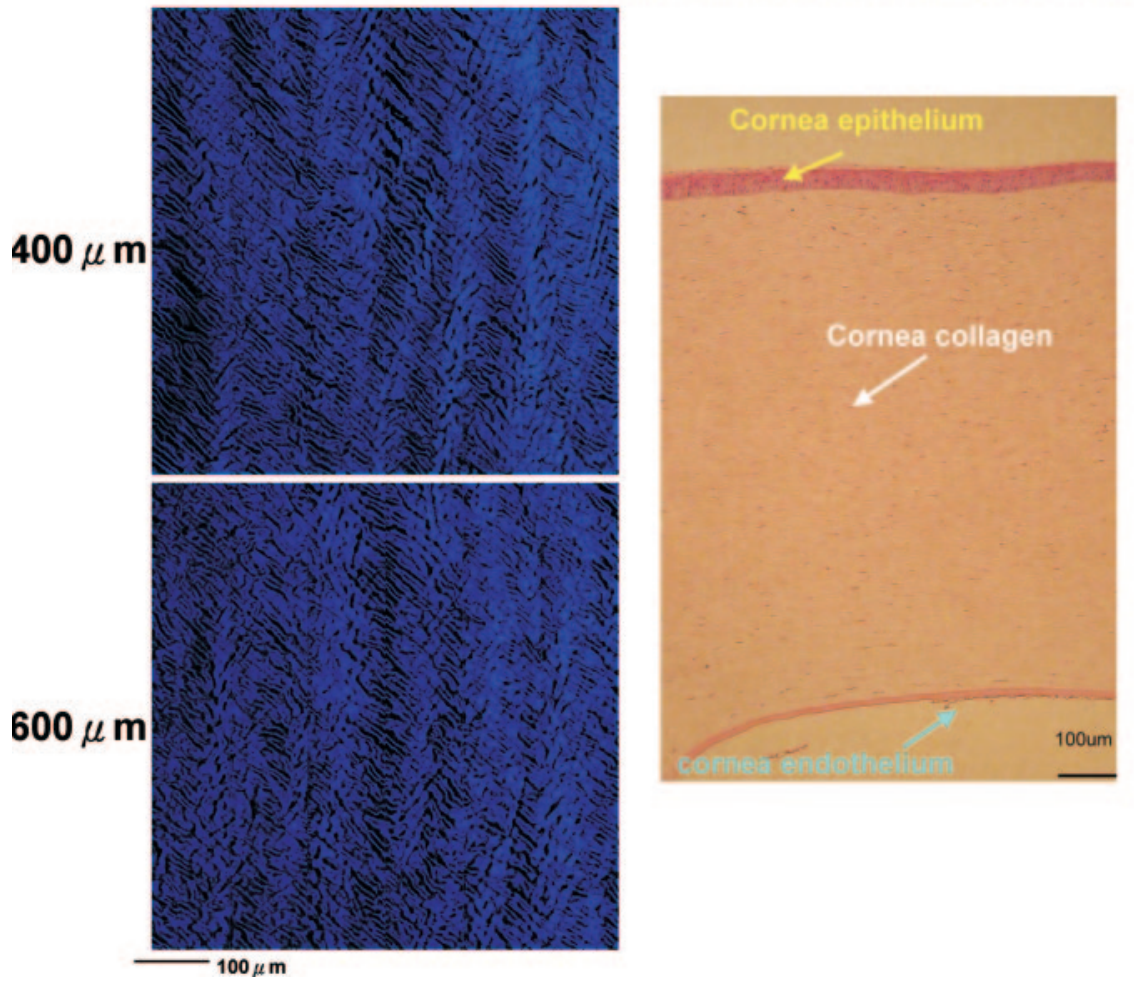

ophthalmology. Before reaching the detectors, the autofluorescence and SHG signals are separated by a secondary dichroic mirror (435DCSX; Chroma Technology). The SHG signal centered at $390 \mathrm{~nm}$ is reflected by the secondary dichroic and further filtered with a band-pass filter (HQ390/20; Chroma Technology), whereas the longer wavelength autofluorescence passes through the dichroic mirror and a broad band-pass filter (E435LP+E700SP; Chroma Technology) before being detected. Both the autofluorescence and SHG signals are detected with single-photon-counting photomultiplier tubes (R7400P; Hamamatsu, Hamamatsu City, Japan). For large-area scans of the eye

specimens, a two-dimensional stage scanning system (H101; Prior Scientific Instruments, Cambridge, UK) was used for specimen translation after each $x-y$ scan of the eye sample. The overlapping multiphoton images acquired in this fashion can then be assembled into a large area, high-resolution tissue map of the eye tissue. An instrument diagram of our experimental apparatus is shown in Figure 1.

\section{Acquisition and Processing of Porcine Eyes}

The porcine eyes used in the study were obtained so that they would be as fresh as possible. The pigs were killed at approximately $4 \mathrm{AM}$, 

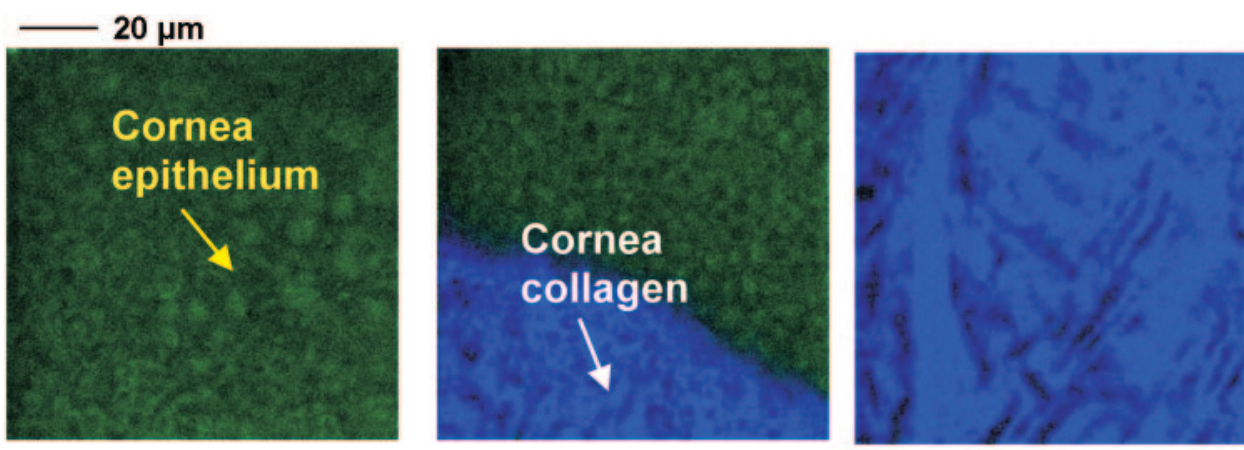

Figure 3. Detailed multiphoton images of a porcine cornea at the depths of 40,70,110,130, 150, and $190 \mu \mathrm{m}$ (left to right; top to bottom). Blue: SHG; green: autofluorescence.
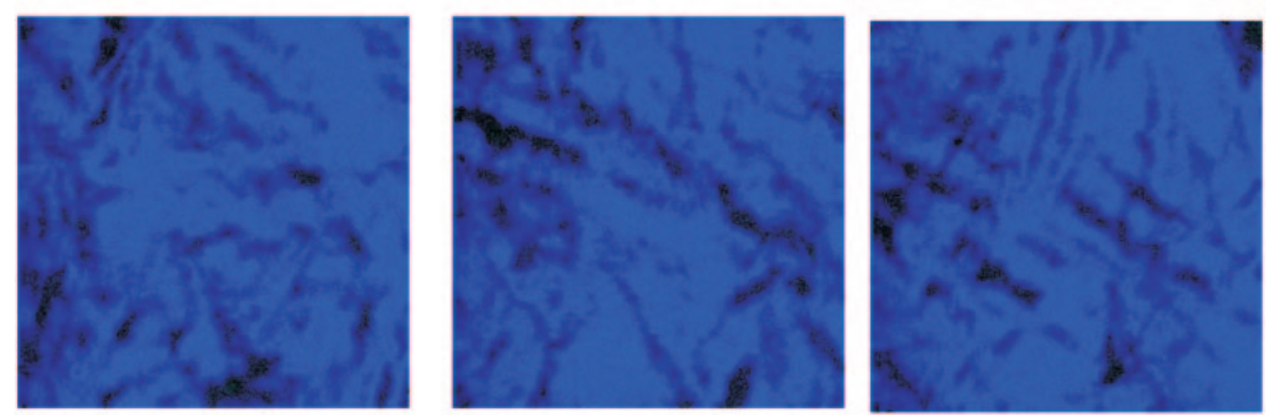

and the eyes were obtained from the local market at approximately 8 AM. Shortly after their acquisition, the porcine eyes were taken to our laboratory. With the extraocular tissue removed, the eyes were then immersed in PBS buffer. For viewing purposes, we placed the eye specimen in an empty coverslip container filled with PBS buffer, and we selected an optically clear porcine eye for multiphoton imaging. The sample was then placed on the microscope stage for viewing. We selected the main regions of the eye exterior for viewing: cornea, limbus, conjunctiva, and sclera. Both localized three-dimensional images and large area scans at different depths were acquired.

We made attempts to acquire the porcine eyes at the earliest possible times. However, the specimens acquired 4 hours after death are not fresh. In an earlier study, it was shown that human donor corneas stored in MEM at $31^{\circ} \mathrm{C}$ under tissue bank conditions undergo endothelial damage over a period of weeks with complete necrosis over 6 weeks. ${ }^{39}$ Nonetheless, in our study, cornea clouding cannot be neglected in all cases, and we selectively chose the porcine eyes with optically clear corneas for imaging purposes. Because our goal is to demonstrate the capability of multiphoton imaging in acquiring spectrally resolved morphologic information on selected ocular tissues, we believe that our approach is acceptable.

After multiphoton imaging was completed, the porcine eyes were placed in fixation medium composed of formalin-PBS (1:9 vol/vol). The eye samples were then dehydrated and embedded in paraffin. Thin sections (approximately $5 \mu \mathrm{m}$ in thickness) of the cornea, limbus, conjunctiva, and sclera were then prepared and labeled with hematoxylin and eosin (HE).

\section{Results}

\section{Cornea}

The multiphoton images of the porcine cornea, along with the histologic images, are shown in Figures 2 and 3, presented in two spatial scales. Displayed in Figure 2 are the large-area multiphoton scans at a fixed position on the cornea surface between the depths of 0 and $862 \mu \mathrm{m}$. The histologic image of the cornea is shown for comparison. Shown in Figure 3 is the detailed three-dimensional scan of the cornea at the depths indicated in the figure legend. At the cornea surface, two sources of sample luminescence helped to identify structural compositions of the tissue. Individual cells in the epithelium could be identified because their cytoplasm was autofluorescent (green), whereas many of their nuclei lacked such signal. Because the porcine eyes we used were structurally intact, the natural curvature of the eye surface was preserved. Therefore, both the cornea surface epithelium (green) and second-harmonic generating collagen fibers (blue) were visible at the same imaging depth. With increasing imaging depths, Figures 2 and 3 show that the source of luminescence became predominantly that of the collagen fibers. The SHG signal from the striated collagen fibers indicates the fiber orientation. Figure 2 also shows the change in direction of fiber orientation with imaging depths. With increasing imaging depths, the SHG intensity due to the collagen fibers decreased, possibly because of the index mismatch-induced spherical aberration between the aqueous medium of the water objective and the cornea. However, at sufficient depths, the autofluorescence signal reappeared. The reappearance of autofluorescence may possibly come from both Descemet's membrane and endothelium. Hence, the contribution of endothelium is not apparent in this figure, which may be related to decay of signal intensity associated with imaging depth. Therefore, we have demonstrated that a combination of SHG and autofluorescence imaging can be used to distinguish cells and collagen fiber orientation throughout the entire cornea. One observation of interest is the variation of collagen fiber packing at different depths. For example, a comparison of the large-area corneal images at 0 and $862 \mu \mathrm{m}$ revealed that the collagen fibers were narrower near the endothelium than at the cornea surface. Whereas our observations of the cornea structural composition agree well with the histologic images, the variations in collagen packing were more pronounced with SHG imaging.

\section{Limbus}

In ophthalmology, the limbus is a region of the eye that attracts considerable attention. Bordering the cornea and the sclera, the limbus has been shown to contain stem cells that proliferate to give rise to epithelial tissues. ${ }^{32,33}$ Therefore, the ability to 


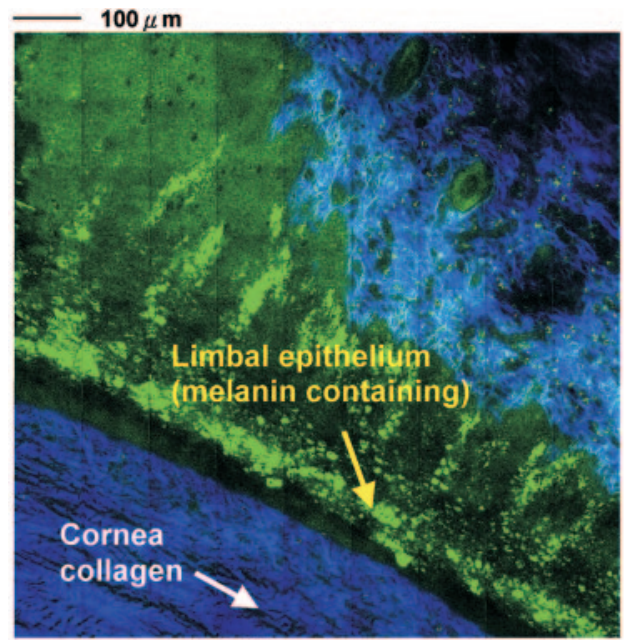

$\mathbf{0} \mu \mathbf{m}$

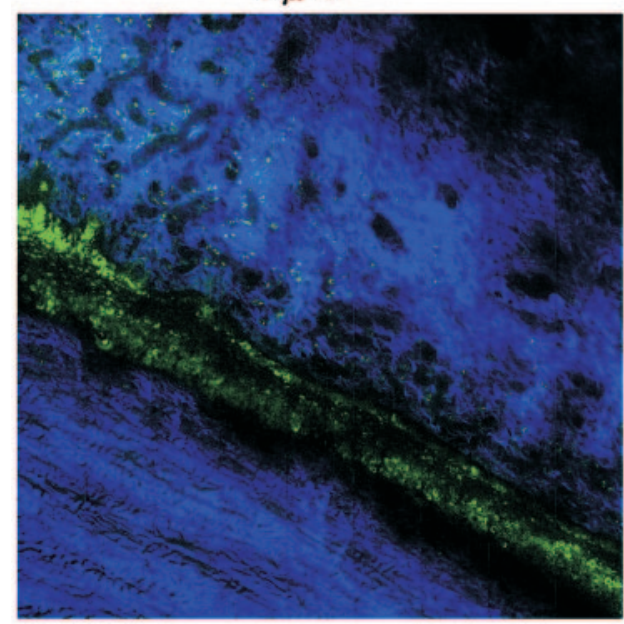

$\mathbf{5 0} \mu \mathbf{m}$

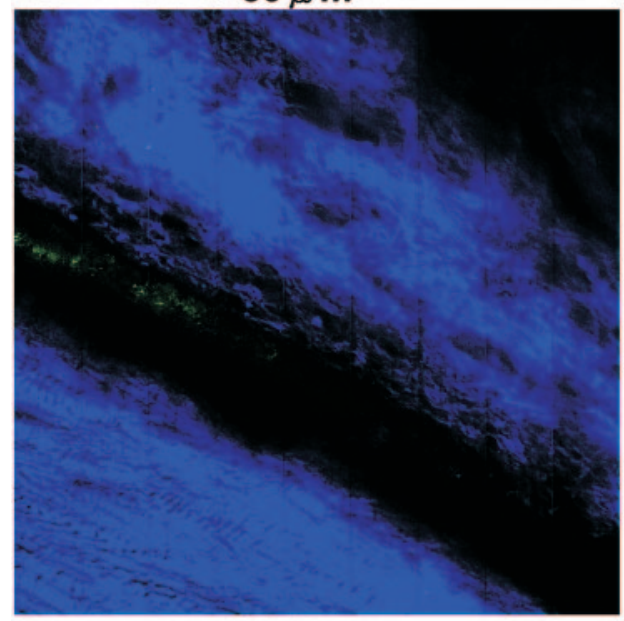

$100 \mu \mathrm{m}$

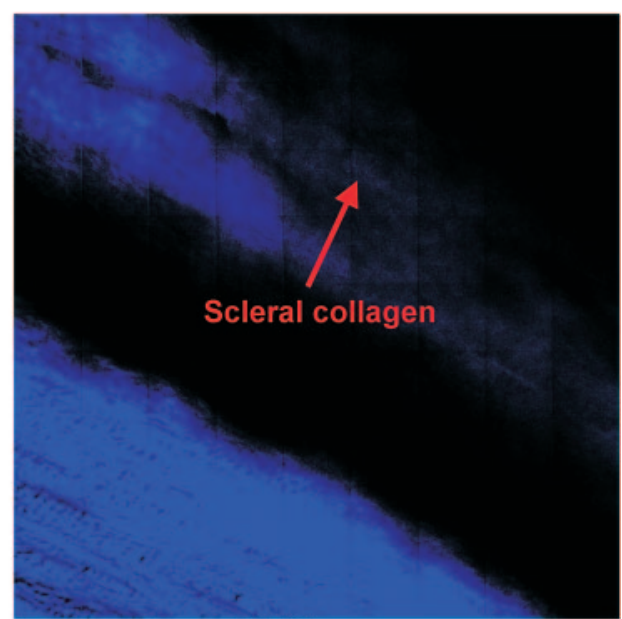

$150 \mu \mathrm{m}$

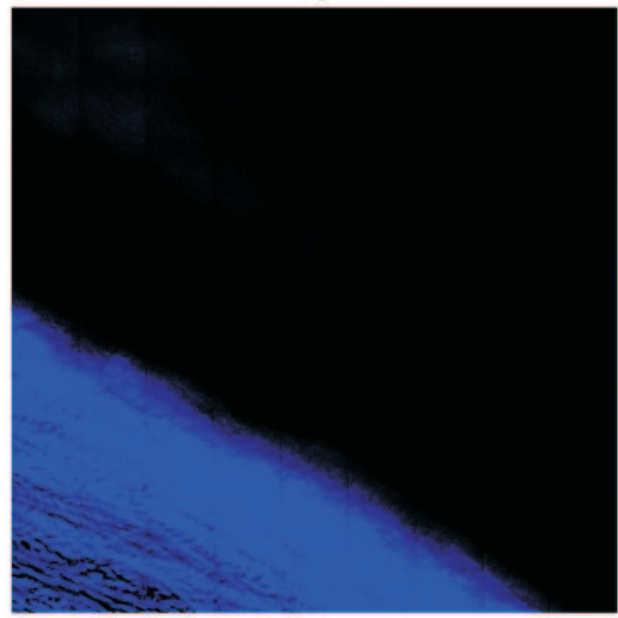

$200 \mu \mathbf{m}$

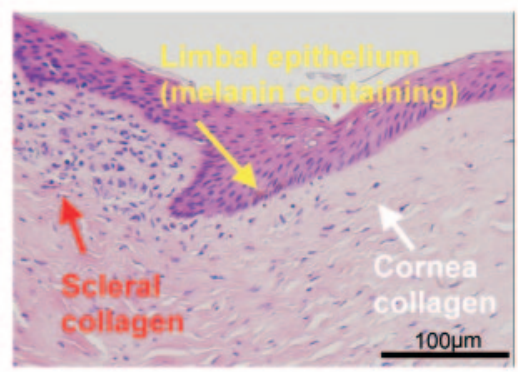

Figure 4. Large-area multiphoton images of a porcine limbus at depths of $0,50,100,150$, and $200 \mu \mathrm{m}$. Bottom right: histologic image. Blue: SHG; green: autofluorescence.

acquire structural information of the limbus noninvasively and monitor the biochemical state of the limbus is of considerable significance in tissue regeneration and engineering of the eye surface. As in the case of the cornea, a combination of multiphoton autofluorescence and SHG imaging was used to identify limbus structures. The large-area multiphoton images at different depths, along with the histologic image, are shown in Figure 4 , and the detailed three-dimensional images of a chosen region are shown in Figure 5. As in the case of cornea imaging, the limbus images demonstrate that multiphoton autofluorescence and SHG imaging reveal features absent from conventional histologic images. Figure 4 shows that the limbal epithelium demonstrates various degrees of autofluorescence. In addition, Figures 4 and 5 illustrate that the cornea and sclera collagen are both capable of generating second harmonic signals. At a deeper imaging depth (nominally at $50 \mu \mathrm{m}$ ), the 

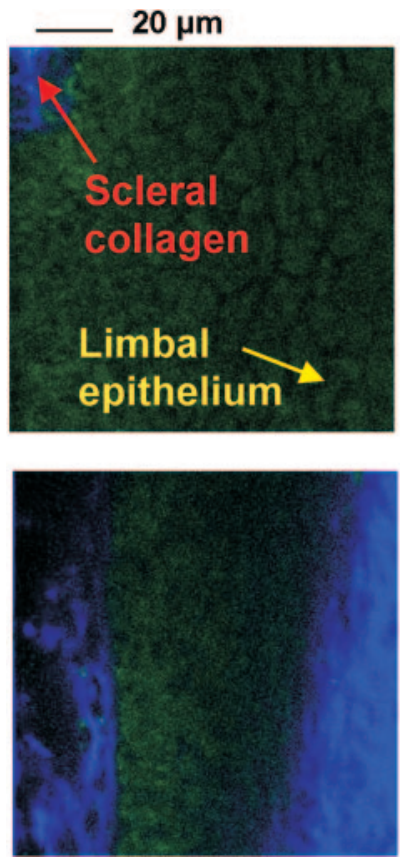
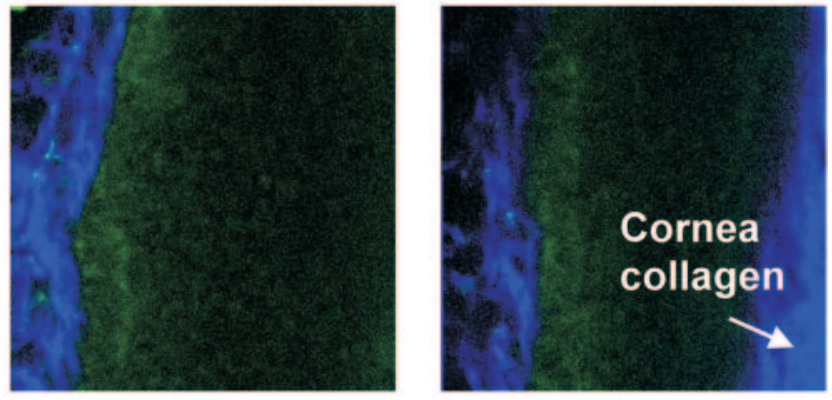

FIGURE 5. Detailed multiphoton images of a porcine limbus at the depths of $0,35,75,85,95$, and 135 $\mu \mathrm{m}$. (left to right; top to bottom) Blue: SHG; green: autofluorescence.
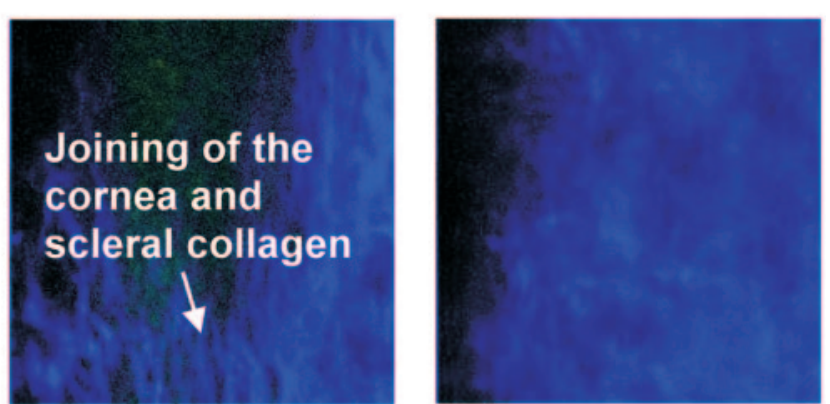

width of the limbus epithelial tissues decreases and the cornea and scleral collagen start to merge. These observations are revealed in greater detail by the multiphoton images in Figure 5 and they agree with our histologic results in Figure 4. Note that in addition to the autofluorescent epithelial cells, the surface large-area scan also reveals the existence of the palisades of Vogt. In our image, the palisades of Vogt can be located by observing the strong autofluorescence emanating from the cells that form a curved lining around the cornea. Furthermore, our results demonstrate that the arrangement of collagen fibers between the cornea and sclera can be sharply distinguished. The corneal side of the eye specimen revealed well-organized packing of the collagen fibers, whereas the collagen fibers within the sclera were less well organized. In fact, a magnified SHG image (Fig. 5) revealed the interface of cornea and sclera collagen fibers at the imaging depth of 95 $\mu \mathrm{m}$. A comparison between the histologic image (Fig. 4) and our data revealed consistency between the two. Our multiphoton images demonstrated the ability to acquire structural information from cells (autofluorescence) and collagen fiber organization (SHG) at the limbus in situ.

\section{Conjunctiva and Sclera}

In addition to imaging the cornea and limbus, we investigated the possibility of applying multiphoton microscopy to reveal morphologic information on the conjunctiva and sclera. As the outer lining of the sclera, the conjunctiva is composed of epithelial cells and connective tissue different from their corneal counterparts. Diseases such as conjunctival melanoma and squamous cell carcinoma are associated with the conjunctiva. In addition, the relatively large area of the conjunctiva make the question of wound healing and drug delivery important. ${ }^{40}$ In comparison, the thermal damage and diseases of the sclera are significant problems in ophthalmology. To be specific, thermotherapy across the sclera can be used for tumor treatment. In addition, minimally invasive multiphoton imaging may be an new method in diagnosing scleritis. ${ }^{38}$ Because the conjunctiva is composed of epithelial cells and loose connective tissue and the sclera of collagen fibers, one would anticipate a combination of multiphoton autofluorescence and SHG imag- ing to offer sharp contrast in distinguishing the conjunctiva from the sclera. As in the case of the cornea and limbus, we acquired multiphoton autofluorescence and SHG images of the porcine conjunctiva and sclera, and the large-area images at different depths and the histologic image are shown in Figure 6. Detailed images of a selected region of the conjunctiva and sclera are shown in Figure 7. As in the case of cornea and limbus imaging, multiphoton autofluorescence is useful in identifying conjunctiva epithelium, and the SHG signal can be used to image sclera collagen. Our results show that with increasing depths, the SHG signal strength decreases rapidly. Unlike the corneal images where the SHG signal can be recorded at the imaging depth of $863 \mu \mathrm{m}$, SHG microscopy penetrated less deeply into the sclera. To be specific, Figure 4 reveals that, although the cornea collagen remained visible at a depth of $200 \mu \mathrm{m}$, the sclera SHG intensity was sufficiently weak for collagen identification. Therefore, the potential of multiphoton diagnosis of sclera diseases may be limited to the imaging depth of a few hundred micrometers. In the large-area images in Figure 6, the SHG signal from the sclera had weakened considerably at a depth of $150 \mu \mathrm{m}$. Therefore, although multiphoton microscopy in the form of SHG imaging is effective in imaging cornea collagen to almost $1 \mathrm{~mm}$ in depth, it is only effective in imaging scleral collagen up to a depth of approximately $200 \mu \mathrm{m}$.

\section{Discussion}

In this work, we used the combined imaging modalities of multiphoton autofluorescence and SHG microscopy to acquire minimally invasive morphologic information on four important constituents of the ex vivo porcine ocular surface: cornea, limbus, conjunctiva, and sclera. We demonstrated that autofluorescence imaging can be used to identify cellular components of the cornea, limbus, and conjunctiva, whereas SHG is useful in imaging corneal and scleral collagen fibers. We also showed that cornea collagen fibers are arranged in an organized fashion with a depth-dependent variation in fiber packing. In comparison, the sclera collagen fibers are more randomly packed and lack the structural organization of their 


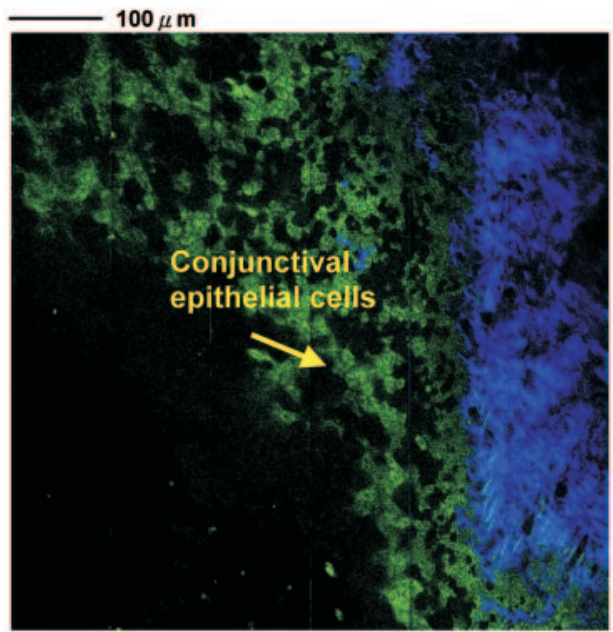

$\mathbf{0} \mu \mathbf{m}$

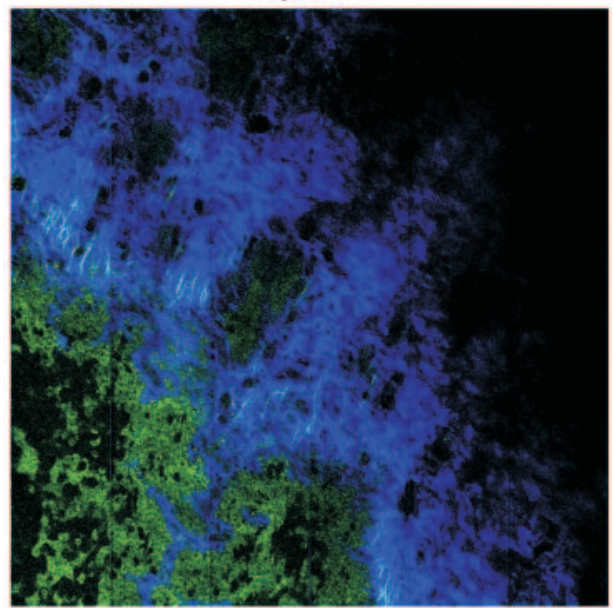

$50 \mu \mathbf{m}$

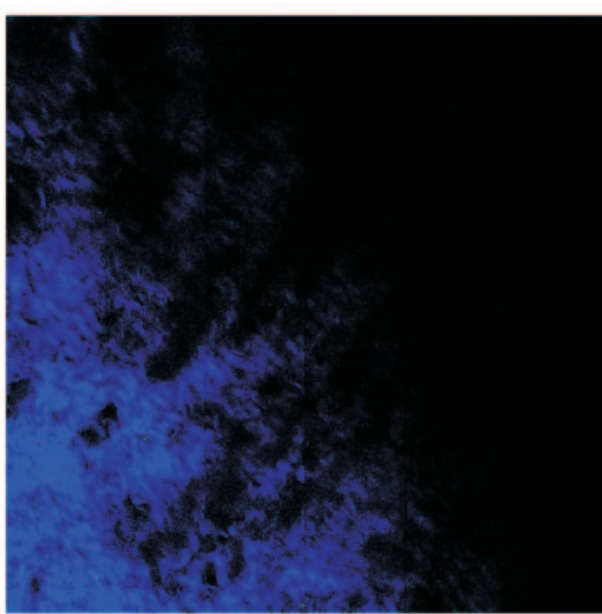

$100 \mu \mathrm{m}$

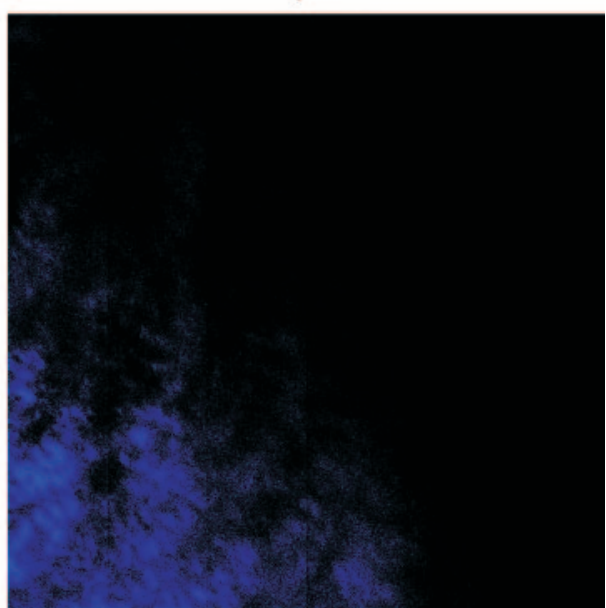

$150 \mu \mathrm{m}$

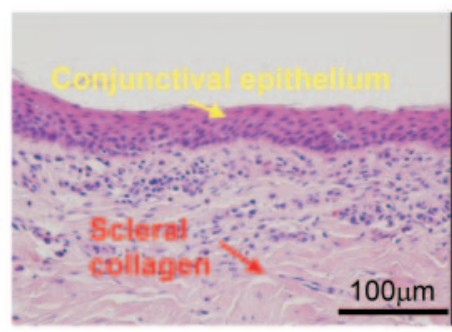

FIGURE 6. Large-area multiphoton images of porcine conjunctiva and sclera. Right: magnified multiphoton image obtained at $0,50,100$, and 150 $\mu \mathrm{m}$. Bottom: histologic image. Blue: SHG; green: autofluorescence. corneal counterparts. Although we were able to image through the cornea, the achievable imaging depth outside the transparent cornea was approximately $200 \mu \mathrm{m}$. Our study demonstrated the ability to acquire structural information using multiphoton microscopy and that a combination of autofluorescence and SHG microscopy can be invaluable in obtaining morphologic features of the eye in a minimally invasive fashion. Although confocal microscopy can provide morphologic information on the retina, cornea, or collagen I matrix orientations, ${ }^{41-44}$ the autofluorescence and SHG imaging capabilities of multiphoton microscopy can provide high-contrast images of ocular epithelial cells and collagen, respectively. Although reflected confocal microscopy is capable of visualizing morphologic features of the superficial epithelium, basal lamina, nuclei of corneal keratocytes, and nerve bundles, ${ }^{45}$ multiphoton microscopy offers image contrast by a different mechanism. In our results, the autofluorescence from the cy- toplasm of the cells is what allowed us to identify individual cells. In contrast, the spectral specificity of the SHG signal at $390 \mathrm{~nm}$ allowed the identification of the collagen orientation and offered an additional degree of contrast enhancement in the imaging of selected ocular tissues.

The spectral contrast offered by multiphoton autofluorescence and SHG microscopy can be used as an effective ex vivo tool for ophthalmologic biopsy. In addition, the minimally invasive nature of multiphoton microscopy can be further developed for in vivo applications in ophthalmology. In the present study, the use of laser power at $16 \mathrm{~mW}$ did not cause significant tissue damage. In optical tissue imaging, it is the combination of the source wavelength, power, and type of tissue that determine the degree of damage. Our successful observation of the multiphoton effects in selected ocular tissues and the negligible photodamage observed support the fact that the laser source we used interacts with the ocular tissues 

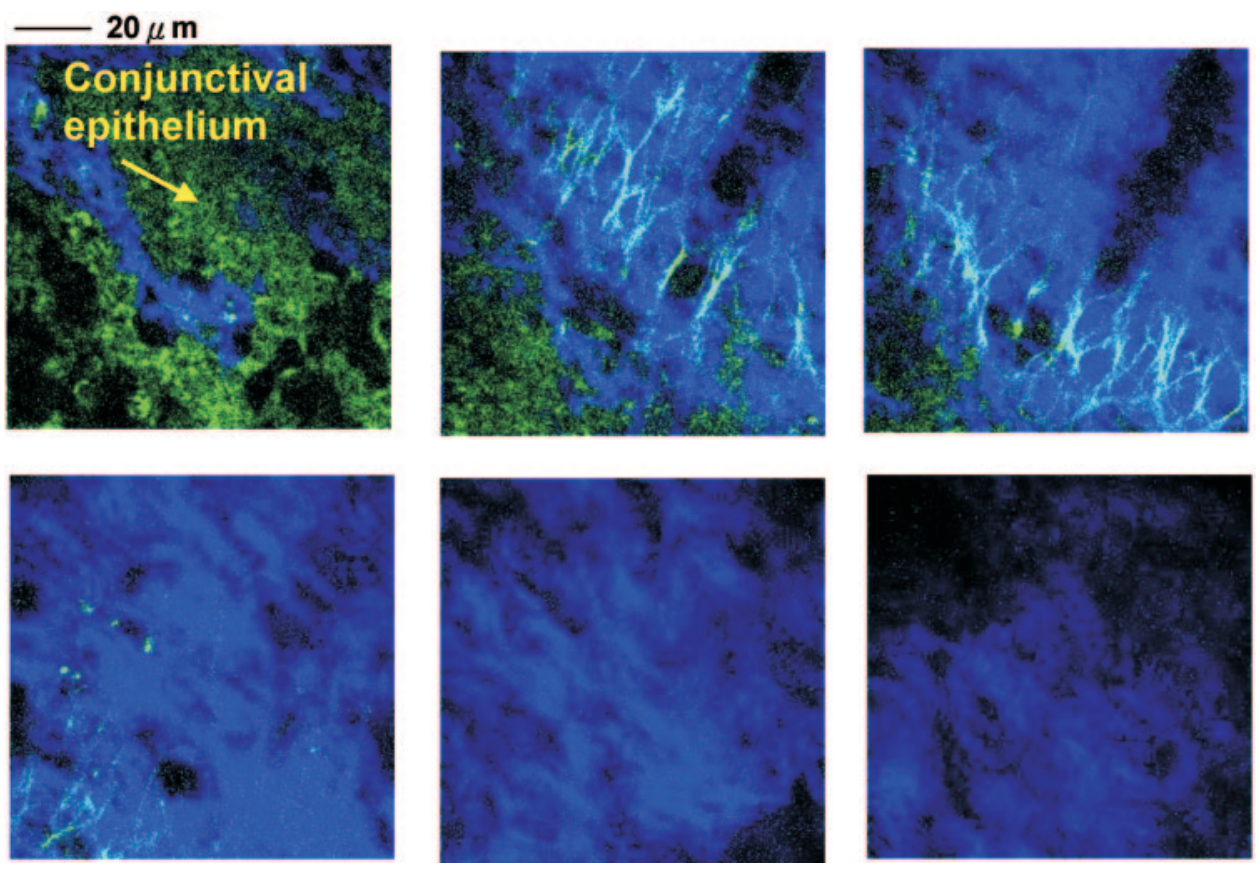

FIGURE 7. Detailed multiphoton images of porcine conjunctiva and sclera at the depths of 50, 65, 70, 80,100 , and $120 \mu \mathrm{m}$ (left to right; top to bottom). Blue: SHG; green: autofluorescence.

studied primarily via multiphoton interactions and that any photodamage incurred would occur primarily in the focal volume. Although additional studies are needed, our study demonstrates the potentials of using multiphoton microscopy for in vivo studies. Furthermore, since an $x-y$ sample stage was used to translate the specimen after each beam-scanning operation for large-area scanning. The development of this technique into an in vivo imaging technique can be facilitated if the focusing objective is designed to translate in selectively imaging the region of the ocular tissue of interest. We envision that the development of a multiphoton, high-speed-scanning, handheld imaging device targeting the selective regions of the ocular surfaces will be a useful addition to the repertoire of imaging technologies currently available for the examination of ocular tissues.

\section{References}

1. Ross MH, Kaye GI, Pawlina W. Histology. 4th ed. Baltimore, MD: Lippincott Williams \& Wilkins; 2003:788-810.

2. Huang D, Swanson EA, Lin CP, et al. Optical coherence tomography. Science. 1991;254:1178-1181.

3. Fujimoto JG, Pitris C, Boppart SA, Brezinski ME. Optical coherence tomography: an emerging technology for biomedical imaging and optical biopsy. Neoplasia. 2000;2:9-25.

4. Fercher AF, Drexler W, Hitzenberger CK, Lasser T. Optical coherence tomography: principles and applications. Rep Prog Phys. 2003;66:239-303.

5. Cavanagh HD, Petroll WM, Alizadeh H, He YG, Mcculley JP, Jester JV. Clinical and diagnostic use of in-vivo confocal microscopy in patients with corneal disease. Ophthalmology. 1993;100:14441454.

6. Masters BR, Bohnke M. Three-dimensional confocal microscopy of the living human eye. Annu Rev Biomed Eng. 2002;4:69-91.

7. Denk W, Strickler JH, Webb WW. 2-Photon laser scanning fluorescence microscopy. Science. 1990;248:73-76.

8. So PTC, Dong CY, Masters BR, Berland KM. Two-photon excitation fluorescence microscopy. Annu Rev Biomed Eng. 2000;2:399429.

9. Denk W, Sugimori M, Llinas R. 2 Types of calcium response Limited to single spines in cerebellar Purkinje-cells. Proc Natl Acad Sci USA. 1995;92:8279-8282.
10. Squirrell JM, Wokosin DL, White JG, Bavister BD. Long-term twophoton fluorescence imaging of mammalian embryos without compromising viability. Nat Biotechnol. 1999;17:763-767.

11. So PTC, Kim H, Kochevar IE. Two-photon deep tissue ex vivo imaging of mouse dermal and subcutaneous structures. Opt Express. 1998;3:339-350.

12. Miller MJ, Wei SH, Parker I, Cahalan MD. Two-photon imaging of lymphocyte motility and antigen response in intact lymph node. Science. 2002;296:1869-1873.

13. Brown E, McKee T, diTomaso E, et al. Dynamic imaging of collagen and its modulation in tumors in vivo using second-harmonic generation. Nat Med. 2003;9:796-800.

14. Yu B, Dong CY, So PTC, Blankschtein D, Langer R. In vitro visualization and quantification of oleic acid induced changes in transdermal transport using two-photon fluorescence microscopy. J Invest Dermatol. 2001;117:16-25.

15. Sun Y, Su JW, Lo W, Lin SJ, Jee SH, Dong CY. Multiphoton polarization imaging of the stratum corneum and the dermis in ex-vivo human skin. Opt Express. 2003;11:3377-3384.

16. Sun Y, Lo W, Lin SJ, Jee SH, Dong CY. Multiphoton polarization and generalized polarization microscopy reveal oleic-acid-induced structural changes in intercellular lipid layers of the skin. Opt Lett. 2004;29:2013-2015.

17. Lee HS, Liu Y, Chen HC, et al. Optical biopsy of liver fibrosis by use of multiphoton microscopy. Opt Lett. 2004;29:2614-2616.

18. Campagnola PJ, Loew LM. Second-harmonic imaging microscopy for visualizing biomolecular arrays in cells, tissues and organisms. Nat Biotechnol. 2003;21:1356-1360.

19. Zipfel WR, Williams RM, Christie R, Nikitin AY, Hyman BT, Webb WW. Live tissue intrinsic emission microscopy using multiphotonexcited native fluorescence and second harmonic generation. Proc Natl Acad Sci USA. 2003;100:7075-7080.

20. Zoumi A, Yeh A, Tromberg BJ. Imaging cells and extracellular matrix in vivo by using second-harmonic generation and twophoton excited fluorescence. Proc Natl Acad Sci USA. 2002;99: 11014-11019.

21. Piston DW, Masters BR, Webb WW. 3-Dimensionally resolved $\mathrm{Nad}(\mathrm{P}) \mathrm{H}$ cellular metabolic redox imaging of the in-situ cornea with 2-photon excitation laser-scanning microscopy. J Microsc (Oxford). 1995;178:20-27.

22. Yeh AT, Nassif N, Zoumi A, Tromberg BJ. Selective corneal imaging using combined second-harmonic generation and two-photon excited fluorescence. Opt Lett. 2002;27:2082-2084. 
23. McDonald MB, Hersh PS, Manche EE, Maloney RK, Davidorf J Sabry M. Conductive keratoplasty for the correction of low to moderate hyperopia: US clinical trial 1-year results on 355 eyes. Ophthalmology. 2002;109:1978-1989.

24. Brinkmann R, Radt B, Flamm C, Kampmeier J, Koop N, Birngruber $\mathrm{R}$. Influence of temperature and time on thermally induced forces in corneal collagen and the effect on laser thermokeratoplasty. $J$ Cataract Refr Surg. 2000;26:744-754.

25. Koch DD, Kohnen T, Anderson JA, et al. Histologic changes and wound healing response following 10-pulse noncontact holmium: YAG laser thermal keratoplasty. J Refract Surg. 1996;12:623-634.

26. Kohnen T, Husain SE, Koch DD. Corneal topographic changes after noncontact holmium: YAG laser thermal keratoplasty to correct hyperopia. J Cataract Refract Surg. 1996;22:427- 435.

27. AsiyoVogel MN, Brinkmann R, Notbohm H, Eggers R, Lubatschowski H, Laqua H. Histologic analysis of thermal effects of laser thermokeratoplasty and corneal ablation using sirius-red polarization microscopy. J Cataract Refract Surg. 1997;23:515-526.

28. Aghamohammadzadeh $\mathrm{H}$, Newton RH, Meek KM. X-ray scattering used to map the preferred collagen orientation in the human cornea and limbus. Structure. 2004;12:249-256.

29. Boote C, Dennis S, Meek K. Spatial mapping of collagen fibril organisation in primate cornea: an X-ray diffraction investigation. $J$ Struct Biol. 2004;146:359-367.

30. Meek KM, Boote C. The organization of collagen in the corneal stroma. Exp Eye Res. 2004;78:503-512.

31. Muller LJ, Pels E, Schurmans LRHM, Vrensen GFJM. A new threedimensional model of the organization of proteoglycans and collagen fibrils in the human corneal stroma. Exp Eye Res. 2004;78: 493-501.

32. Dua HS, Azuara-Blanco A. Limbal stem cells of the corneal epithelium. Surv Ophthalmol. 2000;44:415-425.

33. Wolosin JM, Xiong XL, Schutte M, Stegman Z, Tieng A. Stem cells and differentiation stages in the limbo-corneal epithelium. Prog Retin Eye Res. 2000;19:223-255.
34. Rem AI, Oosterhuis JA, Journee-De Korver HG, Van den Berg TJTP, Keunen JEE. Temperature dependence of thermal damage to the sclera: exploring the heat tolerance of the sclera for transscleral thermotherapy. Exp Eye Res. 2001;72:153-162.

35. Lin SJ, Sun Y, Lo W, Jee SH, Dong CY. Monitoring the thermally induced structural transitions of collagen using second harmonic generation microscopy Opt Lett. 2005;30:622-624.

36. Van Schaik HJ, Alkemade C, Swart W, Van Best JA Autofluorescence of the diabetic and healthy human cornea in vivo at different excitation wavelengths. Exp Eye Res. 1999;68:1-8.

37. Shields CL, Shields JA. Tumors of the conjunctiva and cornea. Surv Ophthalmol. 2004;49:3-24.

38. Watson PG, Young RD. Scleral structure, organisation and disease: a review. Exp Eye Res. 2004;78:609-623.

39. Redbrake C, Salla S, Frantz A. Changes in human donor corneas preserved for longer than 4 weeks. Cornea. 1998;17:62-65.

40. Tasman W, Jaeger EA. Duane's Clinical Ophthalmology on CDROM 2004. Baltimore: Lippincott Williams \& Wilkins; 2004.

41. Guan K, Hudson C, Flanagan JG. Comparison of Heidelberg Retina Tomograph II and retinal thickness analyzer in the assessment of diabetic macular edema. Invest Ophthalmol Vis Sci. 2004;45:610 616.

42. Leduc C, Dupas B, Ott-Benoist AC, Baudouin C. Advantages of the in vivo HRT2 corneal confocal microscope for investigation of the ocular surface epithelium. J Fr Ophtalmol. 2004;27:978-986.

43. Kovoor TA, Kim AS, McCulley JP, et al. Evaluation of the corneal effects of topical ophthalmic fluoroquinolones using in vivo confocal microscopy. Eye Contact Lens. 2004;30:90-94.

44. Petroll WM, Cavanagh HW, Jester JV. Dynamic three-dimensional visualization of collagen matrix remodeling and cytoskeletal organization in living corneal fibroblasts. Scanning. 2004;26:1-10.

45. Jester JV, Petroll WM, Cavanagh HD. Corneal stromal wound healing in refractive surgery: the role of myofibroblasts. Prog Retin Eye Res. 1999;18:311-356. 